\title{
CONFORMAL SPHERICAL PARAMETRIZATION FOR HIGH GENUS SURFACES
}

\author{
WEI ZENG* ${ }^{*}$ XIN LI ${ }^{\dagger}$, SHING-TUNG YAU ${ }^{\ddagger}$, AND XIANFENG GU§
}

\begin{abstract}
Surface parameterization establishes bijective maps from a surface onto a topologically equivalent standard domain. It is well known that the spherical parameterization is limited to genus-zero surfaces. In this work, we design a new parameter domain, two-layered sphere, and present a framework for mapping high genus surfaces onto sphere. This setup allows us to transfer the existing applications based on general spherical parameterization to the field of high genus surfaces, such as remeshing, consistent parameterization, shape analysis, and so on.

Our method is based on Riemann surface theory. We construct meromorphic functions on surfaces: for genus one surfaces, we apply Weierstrass P-functions; for high genus surfaces, we compute the quotient between two holomorphic one-forms.

Our method of spherical parameterization is theoretically sound and practically efficient. It makes the subsequent applications on high genus surfaces very promising.
\end{abstract}

Key words: Conformal Spherical Parameterization, Meromorphic Function, High Genus Surface, Layered Sphere

1. Introduction. Surface parameterization (for a recent survey, we refer the reader to [Floater and Hormann 2005]) is a fundamental tool in computer graphics and benefits many digital geometry processing applications such as texture mapping, shape analysis, compression, morphing, remeshing, etc. Some problems become much easier to deal with a uniform parameter domain. Usually in these settings surfaces are represented as triangle meshes, and the maps are required to be at least no-foldovers and low-distortion in terms of area, angle, or both aspects.

In graphics, spherical parameterizations for genus zero closed surfaces have been proposed and widely used in the past. Most methods [Gotsman et al. 2003; Gu et al. 2004; Haker et al. 2000; Sheffer et al. 2004; Praun and Hoppe 2003] are to directly map the mesh to spherical domain, which is usually formulated as a spherical energy minimization problem, such as conformal, Tutte, Dirichlet, area, spring, stretch energies, or their combinations, as cited in [Floater and Hormann 2005]. The optimization process is to relax the initial map to reach no-foldovers under specified distortion metric.

*Institute of Computing Technology, Chinese Academy of Sciences; Graduate school of the Chinese Academy of Sciences, Chinese Academy of Sciences; Stony Brook University. E-mail: zengwei@cs.sunysb.edu

†Stony Brook University, E-mail: xinli@cs.sunysb.edu

${ }_{\ddagger}^{\ddagger}$ Harvard University. E-mail: yau@math.harvard.edu

§Stony Brook University. E-mail: gu@cs.sunysb.edu 
In medical imaging, spherical parameterizations are broadly applied for brain cortex surface mapping. In this setting, preservation of local shapes are crucial. Therefore, different conformal spherical parameterizations are proposed. Angenent et.al. [Angenent et al. 1999] construct meromorphic functions on the brain surface directly, then lift the mapping onto the sphere using inverse stereographic projections. Gu et.al. [Gu et al. 2004] compute harmonic maps between the brain cortex surface and the unit sphere and use Möbius transformation to adjust the map. Stephenson [Stephenson 2005] uses circle packing method to construct conformal brain mapping.

However, it is well known that the spherical parameterization is limited to genuszero models. To the best of our knowledge, there are few works on high genus surfaces. Recently, Lee et.al. [Lee et al. 2006] present a construction method by Boolean operations of positive and negative spheres. This method requires a lot of interactive human recognitions and geometry editing techniques. Furthermore, the results are not conformal.

In this work, we aims at automatic generalizing conformal spherical parameterizations for high genus surfaces. Because high genus surfaces and spheres are not topologically equivalent, we allow the existence of branch points.

Our method relies on the conformal structure for higher genus meshes. There are two ways to compute conformal structures of general surfaces: one method is based on Hodge theory [Gu and Yau 2002], and the other on discrete surface Ricci flow [Gu et al. 2005, Jin et al. 2006a, Jin et al. 2006b].

According to Riemann surface theory, a conformal map between a surface and the sphere is equivalent to a meromorphic function defined on the surface. The map wraps the surface onto the sphere by several layers and has several branch points, the number of layers and the branch points are determined by the topology of the surface (by Riemann-Hurwitz theorem). The key is how to construct the meromorphic functions on the input surface. For genus one closed surfaces, we construct the wellknown Weierstrass P-function. For high genus surfaces, the quotient between two holomorphic 1-forms is a meromorphic function.

Compared with the existing planar parameterization for high genus meshes, the layered sphere is more natural domain than the planar domain. Employing the properties of sphere geometry and the existing spherical parameterization related applications on genus-zero meshes, the spherical parameterization designed for high genus meshes can get more insights on shape analysis, and introduce more possible applications for high genus meshes.

The contributions of this work are briefly as follows:

- To present a novel practical framework to compute conformal spherical parameterizations for general surfaces; 
- To extend the applications of general spherical parameterization onto that of high genus meshes, including re-meshing, morphing, etc;

- To introduce a systematic method to compute meromorphic functions on general Riemann surfaces.

The remainder of the paper is organized as follows: Section 2 illustrates the basic definition and theorem in theory used in this work, Section 3 describes the algorithm flow, and Section 4 shows the experimental results, and discussion on the method. The paper concludes with a conclusion and future work in Section 5.

2. Basic Theory. In this section, we will briefly introduce the basic theories of meromorphic function on Riemann surfaces. We refer readers to [Farkas and Kra 1980] for details.

2.1. Riemann Surfaces. Definition 2.1. Manifold. Suppose $M$ is a topological space, each point has a neighborhood $U_{\alpha}$ and a homeomorphism $\phi_{\alpha}: U_{\alpha} \rightarrow V_{\alpha}$ from $U_{\alpha}$ to an open set $V_{\alpha}$ in $\mathbf{R}^{\mathbf{n}}$. $\left(U_{\alpha}, \phi_{\alpha}\right)$ is called a local chart. If two such neighborhoods $U_{\alpha}, U_{\beta}$ intersect, then the chart transition function

$$
\phi_{\alpha \beta}=\phi_{\beta} \phi_{\alpha}^{-1}: \phi_{\alpha}\left(U_{\alpha} \cap U_{\beta}\right) \rightarrow \phi_{\beta}\left(U_{\beta} \cap U_{\alpha}\right)
$$

is a homeomorphism from one open set of $\mathbf{R}^{\mathbf{n}}$ to another. Then $M$ is a n dimensional manifold, the set of all local charts $\left\{\left(U_{\alpha}, \phi_{\alpha}\right)\right\}$ form an atlas.

DeFINITION 2.2. Holomorphic functions. In complex analysis, a function $f: \mathbb{C} \rightarrow$ $\mathbb{C},(x, y) \rightarrow(u, v)$ is holomorphic, if and only if it satisfies the following RiemannCauchy equation

$$
\frac{\partial u}{\partial x}=\frac{\partial v}{\partial y}, \frac{\partial u}{\partial y}=-\frac{\partial v}{\partial x}
$$

Definition 2.3. Riemann Surfaces. A Riemann surface is a two dimensional manifold with an atlas $\left\{\left(U_{\alpha}, \phi_{\alpha}\right)\right\}$, such that all chart transitions $\phi_{\alpha \beta}$ are holomorphic functions. The atlas is called the conformal atlas, and each local coordinates $\phi_{\alpha}\left(U_{\alpha}\right)$ are called holomorphic coordinates. The maximal conformal atlas is called a conformal structure of the surface.

Definition 2.4. Riemannian Metric. A Riemannian metric on a manifold is a tensor $\mathbf{g}$, which defines an inner product $<,>_{\mathbf{g}}$ on the tangent spaces of the manifold. Suppose $v_{1}, v_{2}$ are two tangent vectors on the tangent space at point $p$, then the angle between them can be calculated as

$$
\theta=\cos ^{-1} \frac{<v_{1}, v_{2}>_{\mathbf{g}}}{\sqrt{<v_{1}, v_{1}>_{\mathbf{g}}} \sqrt{<v_{2}, v_{2}>_{\mathbf{g}}}} .
$$

In the current work, we focus on surfaces embedded in the Euclidean space $\mathbb{R}^{3}$. Therefore, all the surfaces are with induced Euclidean metric g. We require the 


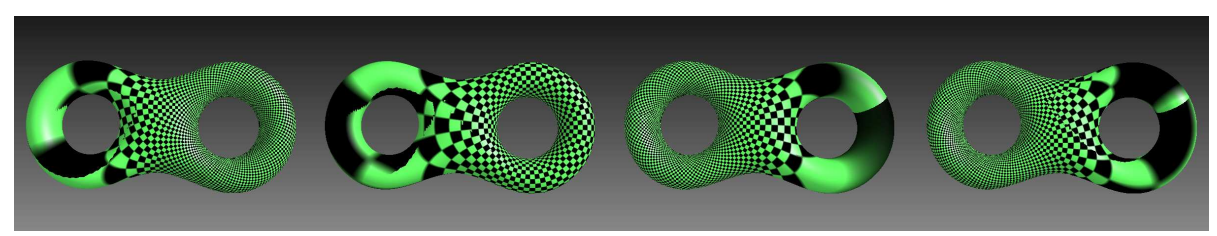

FIG. 1. Example of holomorphic 1-forms. For genus-two cases, there are four 1-forms, described by the check-board texture mapping. The parameter $(u, v)$ of each vertex is the average of 1-forms of its connected edges.

conformal structure and the Riemannian metric be compatible in the following way. Suppose $\left\{\left(U_{\alpha}, \phi_{\alpha}\right)\right\}$ is a conformal atlas, we denote the local coordinates of $\left(U_{\alpha}, \phi_{\alpha}\right)$ as $\left(x_{\alpha}, y_{\beta}\right)$, then the metric tensor is represented

$$
d s^{2}=e^{2 \lambda\left(x_{\alpha}, y_{\alpha}\right)}\left(d x_{\alpha}^{2}+d y_{\alpha}^{2}\right),
$$

where $\lambda\left(x_{\alpha}, y_{\alpha}\right)$ is a function. It can be easily verified that the intersection angle measured by $\mathbf{g}$ equals to that measured by Euclidean metric defined on the parameter domain

$$
d \bar{s}^{2}=d x_{\alpha}^{2}+d y_{\alpha}^{2} .
$$

Namely, the local coordinates preserve angles; therefore conformal structure is also called angle-preserving structure.

2.2. Holomorphic 1-forms. Our algorithm heavily depends on the calculation of holomorphic 1-forms of a given Riemann surface.

Definition 2.5. Holomorphic 1-forms. Given a Riemann surface $X$ with a conformal atlas $\left(U_{\alpha}, z_{\alpha}\right)$, a holomorphic 1-form $\omega$ is defined by a family $\left(U_{\alpha}, z_{\alpha}\right.$, $\omega_{\alpha}$ ), such that (1) $\omega_{\alpha}=f_{\alpha}\left(z_{\alpha}\right) d z_{\alpha}$, where $f_{\alpha}$ is holomorphic on $U_{\alpha}$, and (2) if $z_{\alpha}=$ $\phi_{\alpha \beta}\left(z_{\beta}\right)$ is the coordinate transformation on $U_{\alpha} \bigcap U_{\beta}(\neq \oslash), f_{\alpha}\left(z_{\alpha}\right) \frac{d z_{\alpha}}{d z_{\beta}}=f_{\beta}\left(z_{\beta}\right)$, the local representation of the differential form $\omega$ satisfies the chain rule.

For a Riemann surface $X$ with genus- $g(g>0)$, all holomorphic 1- forms on $X$ form a complex $g$-dimensional vector space ( $2 g$ real dimensions), denoted as $\Omega^{1}(X)$. The quality of a global conformal parameterization for a high genus surface is mainly determined by the choice of the holomorphic 1 -form. The zero points of a holomorphic 1 -form $\omega$ are the points where, on any local representation $\left(U_{\alpha}, z_{\alpha}, \omega_{\alpha}\right), \omega_{\alpha}$ equals zero. For a genus- $g(g>0)$ surface, there are in general $2 g-2$ zero points for each holomorphic 1-form. Figure 1 illustrates the holomorphic 1-forms on a genus-two surface.

2.3. Meromorphic Functions. Definition 2.6. Holomorphic Map. Suppose $X, Y$ are Riemann surfaces with conformal atlases $\left\{\left(U_{\alpha}, \phi_{\alpha}\right)\right\}$ and $\left\{\left(V_{\beta}, \psi_{\beta}\right)\right\}$, a holomorphic map between $X$ and $Y$ is a continuous map $f: X \rightarrow Y$ such that for each 
holomorphic coordinates $\phi_{\alpha}$ on $U_{\alpha}$ containing $x$ on $X$ and $\psi_{\beta}$ defined in a neighborhood of $f(x)$ on $Y$, the composition

$$
\psi_{\beta} \circ f \circ \phi_{\alpha}^{-1}
$$

is holomorphic.

Intuitively, a holomorphic map is a conformal (angle preserving) map between two surfaces. In general, such map doesn't exist between two high genus surfaces. But conformal maps between a high genus to the unit sphere (with branch points) always exist.

Definition 2.7. Meromorphic Function. A meromorphic function $f$ on a Riemann surface $X$ is a holomorphic map to the Riemann sphere $S=\mathbf{C}\{\infty\}$.

The meromorphic function on a Riemann surface usually has multiple branch points. A branch point may be informally thought of as a point at which a "multiplevalued function" changes values when one winds once around it. The neighborhood of branch points wrap around the range a finite or infinite number of times.

Definition 2.8. Branch Point. A branch point of an analytic function is a point in the complex plane whose complex argument can be mapped from a single point in the domain to multiple points in the range.

For example, if $n>1$, then $z^{n}$ has a degree $n$ branch point at 0 .

The relationship between the Euler characteristics of the source surface and the target surface is described by the following theorem, when one is a covering of the other (here, using Riemann sphere) with branch points.

TheOrem 2.9. Riemann-Hurwitz. Let $f: X \rightarrow S$ be a meromorphic function of degree $d$ on a closed connected Riemann surface $X$, and suppose it has branch points $x_{1}, \ldots, x_{n}$ where the local form of $f(x)-f\left(x_{k}\right)$ is a holomorphic function with a zero of multiplicity $m_{k}$. Then

$$
\chi(X)=2 d-\sum_{k=1}^{n}\left(m_{k}-1\right)
$$

where $\chi(X)$ is the Euler number of $X$ and there is $\chi(S)=2$.

In the settings of this paper, the function is two-valued, there are two overlapped layers on the complex plane, which is lifted to be a two-layered sphere by inverse stereo graphic projection, seen in Figure 2. Thus, from the Riemann-Hurwitz formula, the number of branch points can be computed indirectly. Given a closed $g$-holed tori, there are $2 g+2$ branch points (See Figure 3,4 ).

3. Algorithm. This section explains the algorithm for computing the conformal spherical parameterizations for high genus surfaces based on meromorphic functions. 

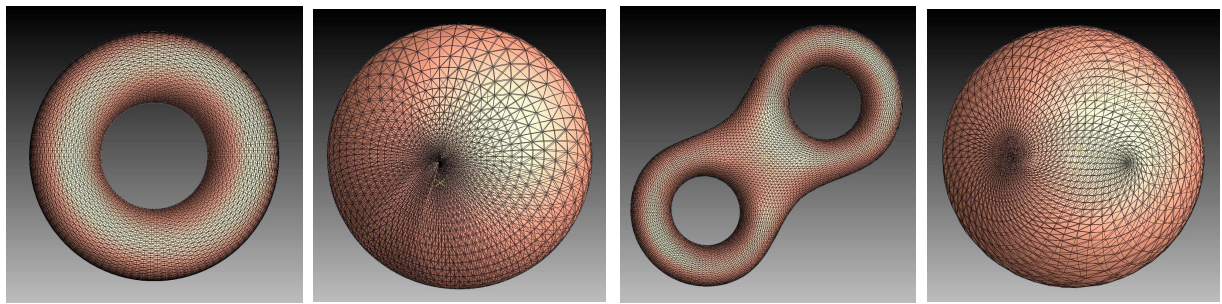

Fig. 2. Two-layered Sphere for genus-one and genus-two cases of our method. From left to right, they are (1) one-hole torus mesh with 10,000 vertices and 20,000 faces, (2) two-layered sphere with four branch points, (3) two-holes torus mesh with 12,286 vertices and 24,576 faces, and (4) two-layered sphere with six branch points. Two layers are connected by branch points where the lines twist together.
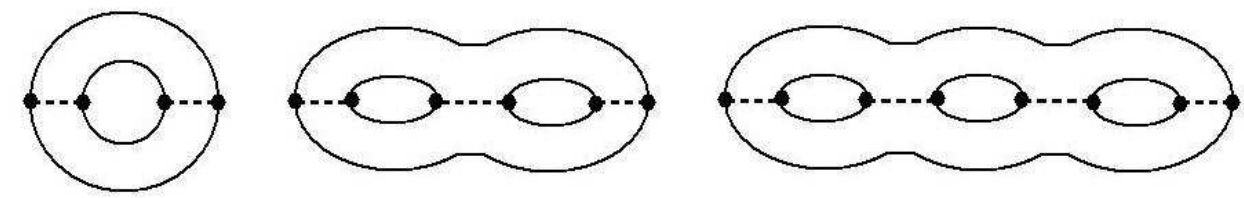

FIG. 3. Branch points illustration for genus- $g(g>0)$ surfaces. There are $2 g+2$ branch points, two for each tunnel. The outer space is regarded as a tunnel here.

3.1. Holmorphic 1-forms. At the first stage, we calculate the basis for the holomorphic 1-form group $\Omega^{1}(M)$. The method is based on the Hodge theory. All the surfaces are represented as triangular meshes (simplicial complex). The followings are the major steps, for details we refer readers to [Gu and Yau 2003]. We assume the input surface is a genus $g$ closed surface.

1. Compute the basis of the first homology group $H_{1}(M, \mathbb{Z}),\left\{\gamma_{1}, \gamma_{2}, \cdots, \gamma_{2 g}\right\}$.

2. Compute the dual cohomology group basis $H^{1}(M, \mathbb{Z}),\left\{\omega_{1}, \omega_{2}, \cdots, \omega_{2 g}\right\}$, such that $\omega_{i}\left(\gamma_{j}\right)=\delta_{j}^{i}$.

3. Diffuse cohomology basis to harmonic 1-forms, such that $\Delta \omega_{i}=0$, where $\Delta$ is the Laplace-Beltrami operator.

4. Compute the conjugate harmonic 1-forms, $\omega_{i}^{*}$, where $*$ is the Hodge star operator. $\left\{\omega_{1}+\sqrt{-1} \omega_{1}^{*}, \omega_{2}+\sqrt{-1} \omega_{2}^{*}, \cdots, \omega_{2 g}+\sqrt{-1} \omega_{2 g}^{*}\right\}$ form a basis for the holomorphic 1-form group.

3.2. Genus One Surfaces. For a genus one closed surface $M$, we first compute the holomorphic 1-form basis, we denote a holomorphic 1-form as $\omega$. $\omega$ induces a Riemannian metric

$$
d s^{2}=\omega \bar{\omega}
$$

which is flat everywhere, namely, the Gaussian curvature induced by $\omega$ is zero. 

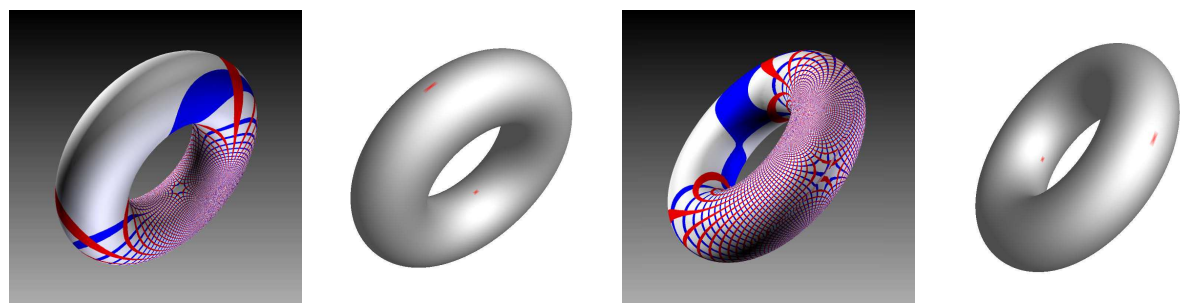

FIG. 4. Branch points of torus. The branch points are shown by the texture, where the region is formed by eight edges. The corresponding location is shown in its right by practical method.

Definition 3.1. Universal Covering Space. Suppose $M$ is a surface, $\pi: \bar{M} \rightarrow M$ is a covering space if for every point $p$ in $M$ there is a neighborhood $U$ of $p$ so that $\pi^{-1}(U)$ is a disjoint union of open sets $V_{i}, i \in I$, and the restriction $\left.\pi\right|_{V_{i}}: V_{i} \rightarrow U$ is a homeomorphism for each $i$. If $\bar{M}$ is simply connected, then $\pi: \bar{M} \rightarrow M$ is a universal covering space.

Definition 3.2. Deck Transformation. Suppose $M$ is a surface, the pair $(\bar{M}, \pi)$ is the universal covering space of $M, \phi: \bar{M} \rightarrow \bar{M}$ is called a deck transformation, if $\pi \circ \phi=\pi$.

All the deck transformations of a surface form a group. For the one-hole torus case, we can embed the universal covering space onto the complex plane using the flat metric. Then the deck transformations of $\left(\bar{M}, d s^{2}\right)$ are translations. We compute the generators of the deck transformation group $\operatorname{Deck}(M)$, which are translations, and denote them as $\left\{w_{1}, w_{2}\right\}$. Any deck transformation can be represented as $w=$ $m w_{1}+n w_{2}, m, n \in \mathbb{Z}$.

The meromorphic function can be easily constructed using the Weierstrass Pfunction on the planar domain. Define

$$
\wp(z)=\frac{1}{z^{2}}+\sum_{w \neq 0}\left(\frac{1}{(z-w)^{2}}-\frac{1}{w^{2}}\right)
$$

where the sum is over all non-zero $w \in \operatorname{Deck}(M)$. Because the sum is essentially over all deck transformations, therefore the Weierstrass function is invariant under the action of $\operatorname{Deck}(M)$,

$$
\wp(z+w)=\wp(z), \forall w \in \operatorname{Deck}(M),
$$

so that this is a meromorphic function defined on the surface $M$. The branch points of $\wp(z)$ are $0, \frac{w_{1}}{2}, \frac{w}{2}, \frac{w_{1}+w_{2}}{2}$, (see Figure 5 and 6 ). The computation example can be seen in Figure 7.

3.3. Genus- $g(g>1)$ Surfaces. Suppose $M$ is a genus $g$ closed surface, $\left\{\omega_{1}, \omega_{2}\right.$, $\left.\cdots, \omega_{2 g}\right\}$ form a basis of the holomorphic 1-form group. A meromorphic function can be constructed using the ratio between two holomorphic 1-forms. 


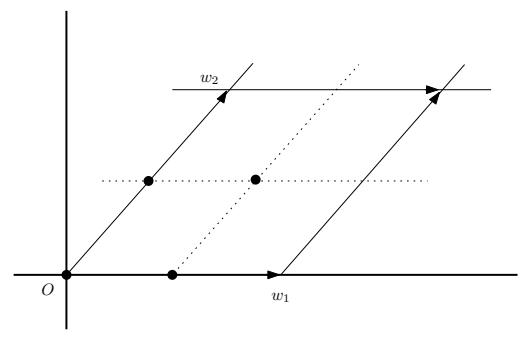

FIG. 5. Generator $\left(w_{1}, w_{2}\right)$ of deck transformations. The branch points here are labeled by four solid circle points, denoting $0, \frac{w_{1}}{2}, \frac{w_{2}}{2}$, and $\frac{w_{1}+w_{2}}{2}$ respectively.
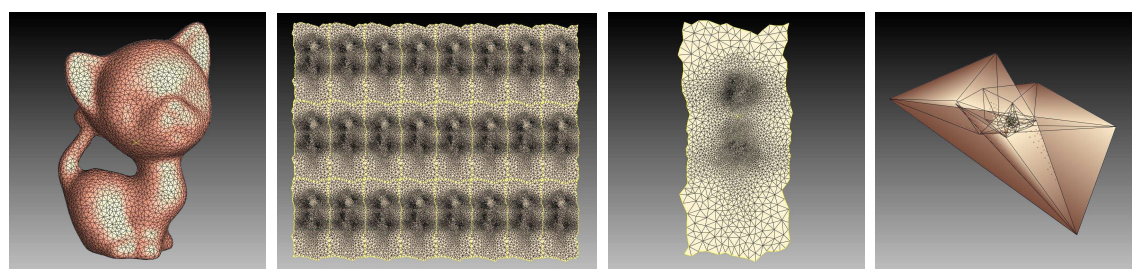

Fig. 6. Universal covering space and Weierstrass P-function. From left to right, they are (1) kitten mesh, (2) part of universal covering space, including 24 parameter periods, (3) one parameter period, and (4) parameter domain under Weierstrass p-function.

Suppose $\tau_{1}$ and $\tau_{2}$ are two holomorphic 1-forms,

$$
\tau_{k}=\sum_{j} a_{k j} \omega_{j}, k=1,2
$$

Suppose $\left\{\left(U_{\alpha}, \phi_{\alpha}\right)\right\}$ is the conformal atlas of the surface $M$, on a local chart $\left(U_{\alpha}, \phi_{\alpha}\right)$,

$$
\tau_{1}=f_{1}\left(z_{\alpha}\right) d z_{\alpha}, \tau_{2}=f_{2}\left(z_{\alpha}\right) d z_{\alpha},
$$

the ratio is

$$
F\left(z_{\alpha}\right)=\frac{f_{1}\left(z_{\alpha}\right)}{f_{2}\left(z_{\alpha}\right)} .
$$

On an overlapping chart $\left(U_{\beta}, \phi_{\beta}\right)$, it can be easily verified that $F\left(z_{\beta}\right)=F\left(z_{\alpha}\left(z_{\beta}\right)\right)$, therefore $F$ is a globally well defined meromorphic function on $M$.

We construct the meromorphic function as $F=\frac{\omega_{1}+\omega_{2}}{\omega_{1}-\omega_{2}}$. The computation example can be seen in Figure 8.

3.4. Branch Point Location. According to Riemann-Hurwitz theorem, meromorphic function on a Riemann surface has branch points, which are important geometric features of the conformal structure of the surface. In practice, it is crucial to accurately locate these branch points.

In order to locate the branch points, we apply the following algorithm to compute the winding number of a closed planar curve. For each point $p \in M$ on the surface, 

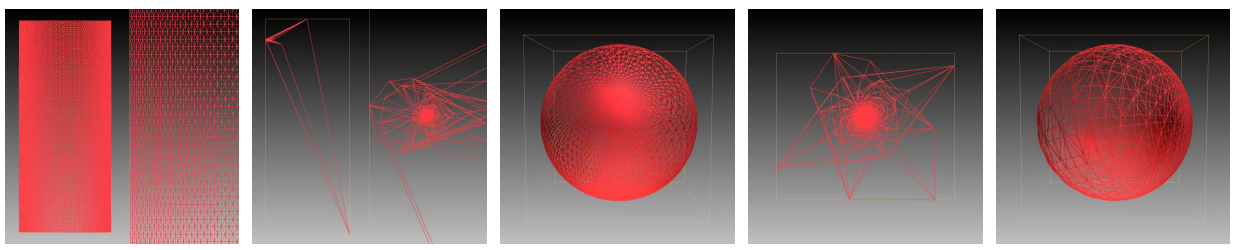

FIG. 7. Genus-one case by Weierstrass p-function. For one-hole torus case, the computation steps are (from left to right) (1) one parameter period with zoomed-in part (right), (2) parameter domain under Weierstrass p-function with zoomed-in part (right), (3) sphere by inverse stereographic projection, (4) parameter domain after Möbius transformation, and (5) sphere of (4) by inverse stereographic projection.
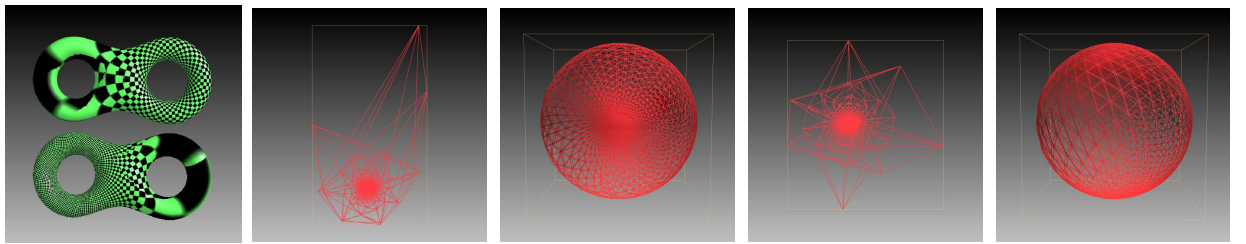

FIG. 8. Genus-n $(n>1)$ case by holomorphic 1-forms. For two-holes torus case, the computation steps are (from left to right) (1) two holomorphic 1-forms $\omega_{1}$ and $\omega_{2}$, (2) parameter domain under quotient function by $\omega_{1}$ and $\omega_{2}$, (3) sphere by inverse stereographic projection, (4) parameter domain after Möbius transformation, and (5) sphere of (4) by inverse stereographic projection.

$F(p) \in \mathbb{C}$ is on the complex plane. We choose a simple closed curve $\gamma$ surrounding $p$ on $M$, then $F(\gamma)$ is a curve on $\mathbb{C}$, we define the winding number as

$$
I(\gamma, p)=\frac{1}{2 \pi \sqrt{-1}} \int_{F(\gamma)} \frac{d z}{z-F(p)} .
$$

If $I(\gamma, p) \neq \pm 1$, then $p$ is a branch point.

In practice, in order to improve the accuracy of computing the winding number, we can zoom the neighborhood of a point $p$ by a Möbius transformation,

$$
\phi: \mathbb{C} \rightarrow \mathbb{C}, \phi(z)=\frac{a z+b}{c z+d}, a, b, c, d \in \mathbb{C}, a d-b c=1.0 .
$$

In order to map the complex plane to the sphere conformally, we use the stereographic projection, suppose a point $(x, y, z)$ is on the unit sphere $\mathbb{S}^{2}$. It is mapped to the planar point $\left(\frac{2 x}{2-z}+\sqrt{-1} \frac{2 y}{2-z}\right)$.

In practice, for different purposes, in order to improve the quality of the spherical conformal surface parameterizations, we can choose the holomorphic 1-forms $\tau_{1}, \tau_{2}$ in Equation 1, and use Möbius transformation to further improve the parameterizations.

4. Results and Discussion. In our implementation, all surfaces are triangular meshes and represented as half edge data structure. The holomorphic 1-forms are 

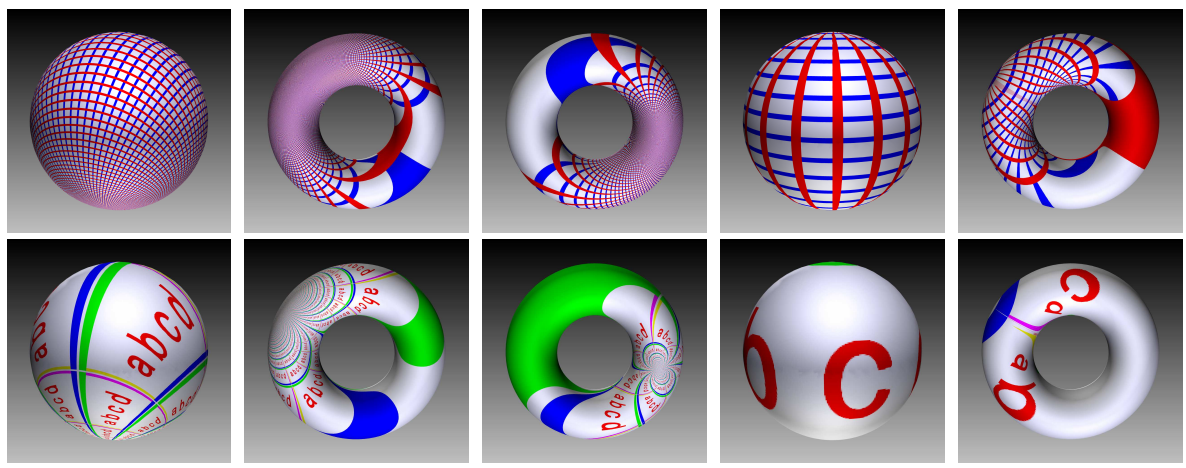

FIG. 9. Spherical conformal parameterization for one-hole torus case. The 1st and 2nd rows are mapped by different textures. From left and to right, each column denotes: (1) conformal parameterization result on layered sphere, (2) initial spherical conformal parameterization result on original surface, (3) spherical conformal parameterization result with Möbius transformation, and (4) curvilinear parameterization of (1), and (5) curvilinear parameterization of (2).
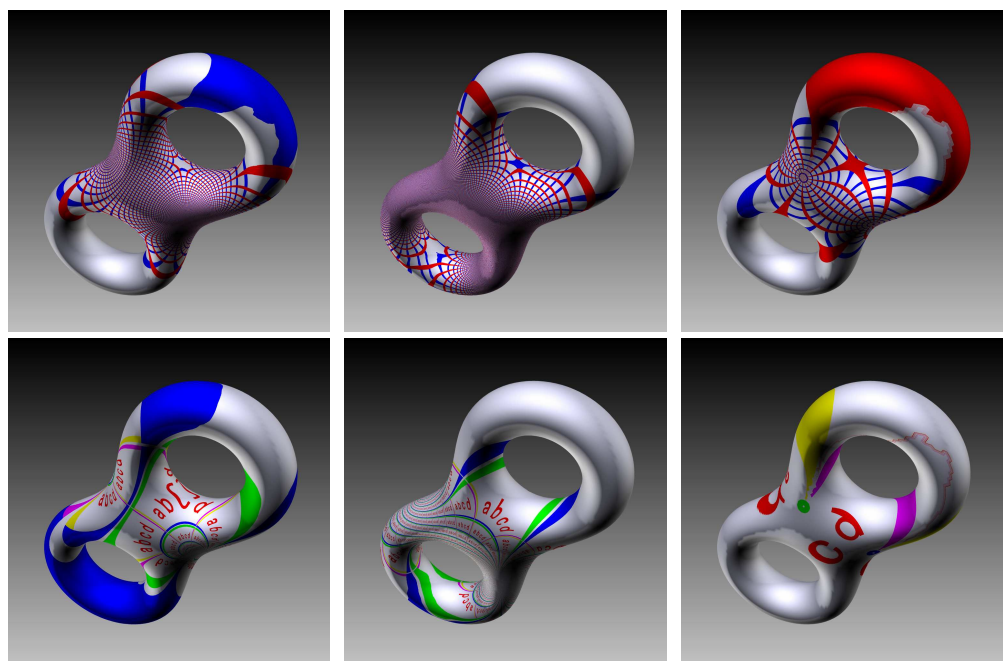

FIG. 10. Spherical conformal parameterization for two-holes torus case. The 1st and 2nd rows are mapped by different textures. From left to right, each column denotes: (1) conformal parameterization result on layered sphere, (2) initial spherical conformal parameterization result on original surface, (3) spherical conformal parameterization result with Möbius transformation, and

represented as simplicial co-chains on the mesh. The whole algorithm is implemented using $\mathrm{c}++$ on windows platform.

Under the theoretical support of meromorphic function, the implementation of our method needs only a simple extension of existing conformal planar mesh parametrization methods[Gu and Yau 2002, Gu and Yau 2003]. The results are bijective maps (except at the branch points) and conformal as illustrated in figures 9, 10, 11, and 12, with colored check-board texture. 

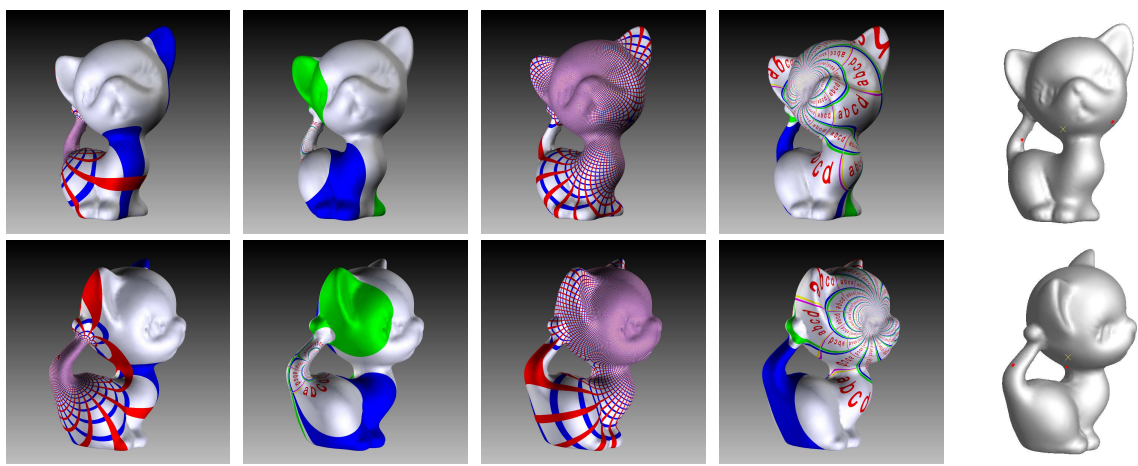

FIG. 11. Spherical conformal parameterization for kitten case with 10,219 vertices and 20,438 faces. The 1st and 2 nd rows illustrate both sides of the model. From left to right, each column denotes: (1) and (2) are initial spherical conformal parameterization results on original surface; (3) and (4) are spherical conformal parameterization results with Möbius transformation; (5) shows the branch points.
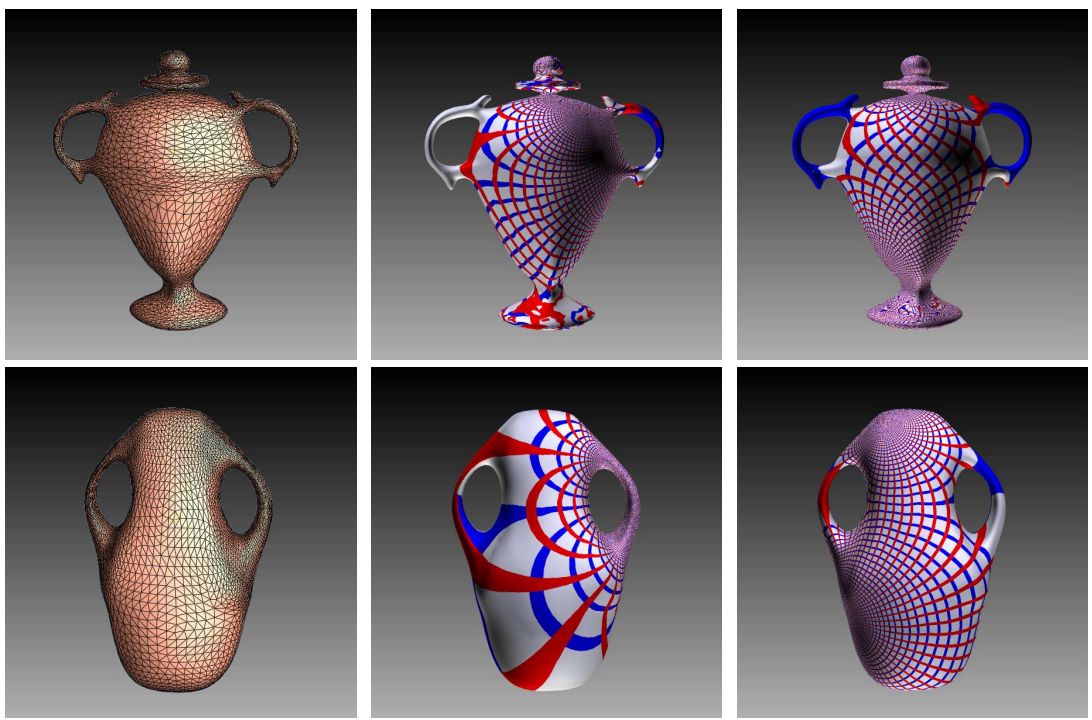

FIG. 12. Spherical conformal parameterization for vase1 and vase2 cases, with the same number vertices and faces. From left to right, each column is (1) triangular meshes with 5,910 vertices and 11,824 faces; (2) initial spherical conformal parameterization results on original surface; (3) the results with Möbius transformation.

We conducted experiments on a variety of meshes. Since our method is based on a composition of several maps, planar parameterization, stereographic projection and conformal relaxation, the validity and conformality of each them guarantees the validity and conformality of the whole map. Using the valid embedding and the periodicity of the planar parameterization, the validity is guaranteed except the overlapping around the branch points. 

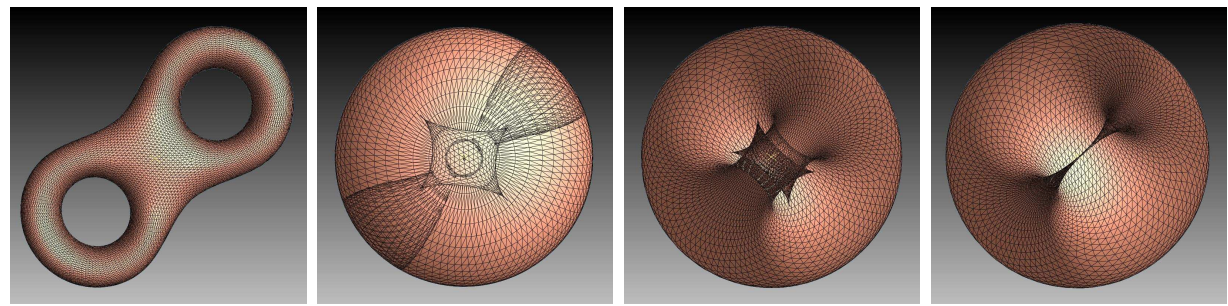

FIG. 13. Gaussian mapping under harmonic optimization with iterative times 0, 500, and 1200.

For high genus surfaces, the branch points can be detected technically or computed from theoretical definition. Their number $b$ is determined by the number $h$ of tunnels. Here, we regard the outer boundary as an outer tunnel. So, for closed two-manifolds, there are formula $h=g+1$, and $b=2 h$. You can easily get this view from that there are two branch points for each tunnel boundary. Figure 4 illustrates the experimental results for genus-one, genus-two surfaces. For genus-one case, their exact locations depend on the start point of computation, because of the periodicity of parameterization domain. Different choices of $\omega_{1}$ and $\omega_{2}$ get different branch points.

From the planar illustration (Figure 3), you can get the insight that the whole surface is split into two parts by the sequence connection of each branch points, labeled in dashed lines. Each part is corresponding to a layer on the spherical domain, which can be seen in Figure 9, where the parameter $(u, v)$ is the spherical curvilinear coordinate $(\theta, \phi)$ [Zayer et al. 2006].

As the most direct application of parameterization, texture mapping results are shown in Figure 9,10, and 11. The conformal parameters $(u, v)$ by stereographic projection may cover a lot of periods of the texture image, so there are many duplicated texture periods.

Compared with Gaussian spherical mapping, our method embeds the high genus surfaces onto sphere with one twisting layer (or multiple layers connecting with branch points) and shows one more natural way for their spherical representations. The simple Gaussian mapping generates the overlapping on handles and can not be embedded properly even under powerfully iterative harmonic relaxation. The handles finally shrink to dense bands, see Figure 13, i.e., the handle information is lost, where under-sampling occurs.

5. Conclusions and Future Work. Conventional conformal spherical parameterizations are limited to genus-zero surfaces. In this work, we generalized conformal spherical parameterizations to surfaces with arbitrary topologies. The proposed method is based on constructing meromorphic functions on Riemann surfaces. High genus surfaces are conformally mapped to the unit sphere with branching points, 
which are the key features for the conformal structure of the surface. The method is theoretically sound and practically efficient.

In the future, we will explore along the following directions:

- Intrinsic triangulations. Triangulate the spherical images with branch points, such that all branch points are vertices, pull back the triangulation to the original surface. This method gives us an intrinsic triangulation, which is solely determined by the conformal structure of the surface.

- Surface matching based on the consistent spherical triangulation. If two surfaces are with similar conformal structures, they can be triangulated in a consistent way using the above intrinsic triangulations. The related applications around consistent parameterization [Praun et al. 2001] can be developed onto high genus surfaces, such as morphing, mapping, matching, etc.

- Meromorphic function based on Poincaré series. Current meromorphic functions on high genus surfaces are the quotient of holomorphic 1-forms, we will develop novel method based on Poincaré series.

\section{REFERENCES}

[Angenent et al. 1999] S. Angenent, S. Haker, A. Tannenbaum, and R. Kikinis, Conformal geometry and brain flattening, in: MICCAI '99: Proceedings of the Second International Conference on Medical Image Computing and Computer-Assisted Intervention, SpringerVerlag, London, UK, 271-278, 1999.

[Asirvatham et al. 2005] A. Asirvatham, E. Praun, And H. Hoppe, Consistent spherical parameterization, in: International Conference on Computational Science (2), 265-272, 2005.

[Farkas and Kra 1980] H. M. FARKAS AND I. KRA, Riemann Surfaces, Springer-Verlag, New York, 1980.

[Floater and Hormann 2005] M. S. Floater And K. Hormann, Surface parameterization: a tutorial and survey, 2005.

[Gotsman et al. 2003] C. Gotsman, X. Gu, And A. Sheffer, Fundamentals of spherical parameterization for $3 d$ meshes, ACM Trans. Graph., 22:3(2003), pp.358-363.

[Gu and Yau 2002] X. GU AND S. YAU, Computing conformal structures of surfaces, in: Communications in Information and Systems, 2(2002), pp. 121-146.

[Gu and Yau 2003] X. Gu AND S. YAU, Global conformal surface parameterization, in: SGP '03: Proceedings of the 2003 Eurographics/ACM SIGGRAPH symposium on Geometry processing, Eurographics Association, Aire-la-Ville, Switzerland, Switzerland, 127-137, 2003.

[Gu et al. 2004] X. Gu, Y. Wang, T. F. Chan, P. M. Thompson, and S.-T. Yau, Genus zero surface conformal mapping and its application to brain surface mapping, IEEE Transaction on Medical Imaging 23, 8(2004), pp. 949-958.

[Gu et al. 2005] X. Gu, F. LuO, AND S.-T. YAU, Tutorial on discrete ricci flow for global parameterizations, Technique report, August, 2005.

[Haker et al. 2000] S. Haker, S. Angenent, A. Tannenbaum, R. Kikinis, G. Sapiro, and M. HALle, Conformal surface parameterization for texture mapping, IEEE Transactions on Visualization and Computer Graphics, 6:2(2000), pp. 181-189.

[Jin et al. 2006a] M. Jin, J. Kim, F. LuO, S. LeE, AND X. Gu, Conformal surface parameterization 
using euclidean ricci flow, Technique report, May, 2006.

[Jin et al. 2006b] M. JiN, F. LUO, AND X. GU, Computing surface hyperbolic structure and real projective structure, in: SPM '06: Proceedings of the 2006 ACM symposium on Solid and physical modeling, ACM Press, New York, NY, USA, 105-116, 2006.

[Kraevoy and Sheffer 2004] V. Kraevoy And A. Sheffer, Cross-parameterization and compatible remeshing of $3 d$ models, ACM Trans. Graph. 23:3(2004), pp. 861-869.

[Lee et al. 2006] T.-Y. Lee, C.-Y. YaO, H.-K. Chu, M.-J. TaI, And C.-C. Chen, Generating genus-n-to-m mesh morphing using spherical parameterization, Computer Animation and Virtual Worlds, 17:3-4(2006), pp. 433-443.

[Praun and Hoppe 2003] E. Praun AND H. Hoppe, Spherical parametrization and remeshing, in: SIGGRAPH '03: ACM SIGGRAPH 2003 Papers, ACM Press, New York, NY, USA, 340349, 2003.

[Praun et al. 2001] E. Praun, W. Sweldens, And P. Schröder, Consistent mesh parameterizations, in: SIGGRAPH 2001, Computer Graphics Proceedings, ACM Press / ACM SIGGRAPH, E. Fiume, Ed., 179-184, 2001.

[Sheffer et al. 2004] A. Sheffer, C. Gotsman, And N. Dyn, Robust spherical parameterization of triangular meshes, Computing, 72:1-2(2004), pp. 185-193.

[Stephenson 2005] K. Stephenson, Introduction to Circle Packing: the Theory of Discrete Analytic Functions, Camb. Univ. Press, New York. ISBN 0-521-82356-0, QA640.7.S74, 2005.

[Zayer et al. 2006] R. ZAYER, C. RössL, AND H.-P. SEIDEL, Curvilinear spherical parameterization, in: Shape Modeling International (SMI), IEEE, Matsushima, Japan, 57-64, 2006. 